\title{
Thermal study of the effect of several solvents on polymerization of acrylonitrile and their subsequent pyrolysis
}

\author{
P.J. Sánchez-Soto ${ }^{a}{ }^{*}$, M.A. Avilés ${ }^{a}$, J.C. del Río ${ }^{b}$, \\ J.M. Ginés ${ }^{\mathrm{c}}$, J. Pascual ${ }^{\mathrm{d}}$, J.L. Pérez-Rodríguez ${ }^{\mathrm{a}}$ \\ a Instituto de Ciencia de Materiales de Sevilla (ICMSE), \\ Centro Mixto Consejo Superior de Investigaciones Cientificas (C.S.I.C.), Universidad de Sevilla, \\ c/Americo Vespucio s/n, Isla de la Cartuja, 41092 Seville, Spain \\ ${ }^{\mathrm{b}}$ Instituto de Recursos Naturales y Agrobiología de Sevilla, C.S.I.C., P.O. Box 1052, \\ 41080 Seville, Spain \\ ${ }^{\mathrm{c}}$ Departamento de Farmacia y Tecnología Farmaceútica, Facultad de Farmacia, Universidad de Sevilla, \\ c/Profesor García González s/n, 41071 Seville, Spain \\ d Member of the Unidad Asociada al C.S.I.C., 'Materiales y Superficies' through ICMSE, \\ Departamento de Ingeniería Civil, de Materiales y Fabricación, ETSII, Universidad de Málaga, \\ 29013 Málaga, Spain
}

Received 17 April 2000; accepted 20 October 2000

\begin{abstract}
The polymerization of acrylonitrile to polyacrylonitrile (PAN) has been studied using several solvents: $N, N$-dimethylformamide (DMF), hexane, toluene, water, and in bulk form (no solvent). The addition of DMF is the only case where both monomer and polymer are soluble in the solvent. Thermal analyses of the resultant products after polymerization have been performed by differential scanning calorimetry and pyrolysis-gas chromatography/ mass spectrometry. The effect of the solvents employed as media for polymerization is interpreted from the results of the thermal and structural (X-ray diffraction) methods. The polymer samples obtained when using water or toluene as solvents have the greater content of amorphous components compared to the others. The amide molecules are difficult to completely eliminate in the product obtained after the polymerization reaction and even after prolonged heating at $110^{\circ} \mathrm{C}$ and remain occluded. DMF can be considered to exert a plasticized effect on PAN and is even capable of forming complexes by dipolar bonding. As
\end{abstract}

* Corresponding author. Tel.: + 34-95-4489527; fax: + 34-95-4460165.

E-mail address: pedroji@cica.es (P.J. Sánchez-Soto). 
a result of this interaction, the thermogram is quite different from the other samples studied in the present work, showing a single sharp exothermic peak. This is associated with nitrile group polymerization (cyclization) of PAN. It is deduced that the amount of heat evolved as well as the temperature interval over which it is released are influenced by the chemical processing of PAN, in particular when using DMF as solvent for both monomer and polymer. Pyrolysis of the different PAN samples revealed the release of occluded solvent molecules, mainly when using DMF, and compounds produced from the thermal degradation processes. Different types of cyclized compounds, such as pyridine derivatives and aromatic nitriles were identified. All these compounds could be derived from cyclized PAN structures which are not completely degraded by the thermal treatment of pyrolysis. Alkyldinitriles have also been tentatively identified associated with the final molecular breakdown of cyclized structures with six-member rings by pyrolysis. Valuable complementary information on the structure of the PAN samples (homopolymer) obtained using the different processing approaches involving several solvent media has been provided by pyrolysis. The present results will improve our understanding of the evolution of the structure and properties of carbon and activated carbon fibres which will enable us to establish processing strategies in order to obtain these materials under adequate and reproducible conditions. (C) 2001 Elsevier Science B.V. All rights reserved.

Keywords: Acrylonitrile; Polyacrylonitrile; N,N-Dimethylformamide; Pyrolysis; Cyclization; Carbon fibres; Pyridine derivatives; Solvents; Processing

\section{Introduction}

Carbon fibres are now important industrially and have gained a wide range of application, from sports equipment to the aerospace industry [1-8]. This has lead to the development of high-strain and high-performance carbon fibres [1,9-19]. They are excellent reinforcers for composites, although their cost is so high that an increase in their consumption can hardly be expected. On the other hand, activated carbon fibres have received increasing attention in recent years as an adsorbent, filter, etc., for filtering automobile gasoline, deodorizing tobacco smoke, recovering solvents and purifying water [20-25]. The advantage of fibrous active carbon, compared to finely powdered active carbon, is the higher bulk volume of the former which can lead to higher adsorption rates because of the more open base structure $[22,23]$. The activated carbon fibres must be processed to remove the adsorbate, which regenerates the adsorbent carbon for subsequent use as an adsorbent material by extraction with different solvents, chemical regeneration or thermal treatments $[21,24,25]$. Further developments have shown that carbon fibres can be useful as functional electrical materials [26,27]. For instance, the preparation of semiconducting carbon fibre by pyrolysis of polyacrylonitrile (PAN) precursor has been described [26]. PAN fibres, with a novel coiled morphology, have been prepared as precursors for semiconducting carbon infrared detection elements [27]. However, a careful control of pyrolysis conditions is required for the preparation of semiconducting carbon fibres. 
Carbon fibres and activated carbon fibres are produced by carbonizing a raw material, such as polyacrylonitrile (PAN) fibre [1-19,22]. Most $(90 \%)$ of the carbon fibres produced worldwide are obtained from PAN and the rest from other raw materials, such as phenolic, rayon or pitch fibres. Although PAN fibres are more expensive than rayon fibres, they are used extensively as a source of carbon fibres because their carbon yield is almost twice that of rayon. Pitch carbon fibres have poor strength properties or non-reproducibility of their properties. PAN fibres have been found to be the most suitable precursors for making high-performance carbon fibre [1-19]. Thus, an important step in preparing carbon fibre from the PAN fibre is to heat the precursor at $200-300^{\circ} \mathrm{C}$ in air or in an oxygen-containing atmosphere, i.e. the stabilization process. This process leads to the formation of a ladder polymer in stabilized fibre. Subsequently, carbonization and graphitization is carried out at higher temperatures in an inert atmosphere to obtain a particular type of carbon fibre. A review has been made by Jain et al. [13,14] concerning the conversion of acrylonitrile-based precursor fibres to carbon fibres, with an emphasis on precursor morphology, thermooxidative stabilization, and the physical and morphological aspects. Stabilized PAN fibres are also used as fibres in their own right for high-temperature applications and for fire-retardant textiles [16]. The modification of PAN fibres is also an interesting way to produce high-performance carbon fibres $[18,19]$.

In the present study, polymerization of acrylonitrile has been studied in bulk form and using several solvents: $N, N$-dimethylformamide (DMF), hexane, toluene, or water. Thermal analyses of the resultant products after polymerization have been performed by differential scanning calorimetry (DSC) and pyrolysis-gas chromatography/mass spectrometry (Py-GC/MS), a powerful tool for the structural analysis of the thermal degradation products of different polymers. X-Ray powder diffraction (XRD) has been used to investigate the structural differences. The effect of the solvents employed as media for polymerization is interpreted from the results of the thermal and structural methods.

\section{Materials and methods}

\subsection{Materials}

Polymerization of acrylonitrile (AN) to polyacrylonitrile (thereafter PAN or the polymer) has been assayed in bulk form (without any solvent) and when using various solvents: DMF, hexane, toluene, methanol or water.

As a general procedure for polymer preparation, $2 \mathrm{ml}$ of acrylonitrile (AN, 99\%, Carlo Erba) and 0.7 wt.\% of benzoyl peroxide (Merck) as polymerization initiator [28], were intensively mixed. The product was then heated in a closed flask at $60^{\circ} \mathrm{C}$ for $24 \mathrm{~h}$ to polymerize the AN. This step was also carried out using a $25 \mathrm{wt} . \%$ solution of AN in hexane, toluene, methanol or DMF. A mixture of $25 \mathrm{wt} . \% \mathrm{AN}$ and deionized water was also used after prior shaking to obtain an emulsion. In all these cases, the products were heated in a closed flask at $60^{\circ} \mathrm{C}$ for $24 \mathrm{~h}$ to 
polymerize the $\mathrm{AN}$, followed by a drying treatment at $110^{\circ} \mathrm{C}$. All the final products were lightly ground using a centrifugal mill (ZSM-1) with knives of stainless steel.

\subsection{Methods}

XRD diagrams were obtained using a Siemens D-501 diffractometer at $26 \mathrm{~mA}$ and $36 \mathrm{kV}$, with Ni-filtered $\mathrm{Cu} \mathrm{K} \alpha$ radiation and a graphite monochromator. A scanning rate of $1^{\circ}(2 \theta)$ was used at the range $2-70^{\circ}(2 \theta)$ with a time constant of $1 \mathrm{~s}$.

DSC curves were obtained using a Mettler equipment, model FP85, by heating from 30 to $400^{\circ} \mathrm{C}$ in air at a heating rate of $10^{\circ} \mathrm{C} \mathrm{min}{ }^{-1}$. Powdered samples $(10 \mathrm{mg}$ each) were weighed and encapsulated in flat-bottomed aluminium pans of $45 \mu \mathrm{l}$ volume with crimped-on lids. Evolved heat was determined following calibration with indium (28.4 $\mathrm{J} \mathrm{g}^{-1}$ ) using the integration of the areas under the endothermal peaks and corrected base lines.

Analytical pyrolysis was carried out at $700^{\circ} \mathrm{C}$ in a $\mathrm{CDS}$ Pyroprobe 190 unit consisting of a Pt coil heated at a rate of $20^{\circ} \mathrm{C} \mathrm{ms}^{-1}$. The sample $(2 \mathrm{mg})$ was placed in a quartz tube using quartz wool for end plugs. The pyrolysis unit was mounted in the injection block of a HP 5988 A GC-MS system. The volatile pyrolysis products were separated on a DB-5 (J\&W Scientific) fused silica capillary column (30 $\mathrm{m} \times 0.25 \mathrm{~mm}$ i.d.). The products were first concentrated in a loop of the column in a liquid nitrogen cold trap, and then the GC oven was heated from 40 to $300^{\circ} \mathrm{C}$ at a rate of $6^{\circ} \mathrm{C} \min ^{-1}$, with a 10 -min final hold. The pyrolysis products were identified by comparing their electron impact mass spectra with mass spectra in libraries, and with the mass spectra and GC retention time of standard compounds.

\section{Results and discussion}

\subsection{Processing approaches}

In the experimental section, the general method was described, and in this section, the results are detailed and discussed. The following were considered: (a) the use of no solvents, (b) solvents where both monomer and polymer are soluble, (c) solvents where monomer is soluble and polymer is not, and (d) solvents where both monomer and polymer are not soluble. AN is a particular case among vinyl monomers regarding the solvent dependence of the propagation rate constant in homogeneous free radical polymerization [11]. This propagation rate constant is considerably higher in water than in other solvents. In every case, 0.7 wt. $\%$ of benzoyl peroxide as the polymerization initiator was added [17,28,29]. The excess liquid AN was always removed, and the product was heated in a closed flask at $60^{\circ} \mathrm{C}$ for $24 \mathrm{~h}$ to avoid liquid evaporation and to polymerize the AN. A subsequent treatment of drying and grinding was carried out, as described in the experimental section. 
The application of the different processing approaches yields the same polymer (PAN), but the medium used influenced the final aspect of this compound. There are advantages and disadvantages of these approaches:

1. Bulk processing. This is the simplest method. The polymerization reaction was carried out without any solvent. After polymerization, a hard compact was obtained with a yellowish-white colour. Moreover, the shape of the flask where this experiment was carried out appeared to be filled by the compacted product. Thus, it was necessary to break the flask in order to recover the sample after the elimination of the glass fragments. A clear advantage of this approach is that the elimination of solvents is avoided.

2. Suspension processing (both monomer and polymer are soluble in the solvent): The solvent medium (DMF) facilitates the reaction. DMF is a solvent of both AN and PAN. Thus, a previous solution (25 wt.\%) of AN in DMF was used. In this processing approach, an increase in viscosity of the liquid and the formation of a dark brown colour as a consequence of polymer formation was observed. The polymer was separated by centrifugation. It was necessary to dry the solution at $110^{\circ} \mathrm{C}$ to remove the liquid. This approach facilitates the handling by the control of the monomer concentration in the solvent (DMF), i.e. the viscosity control. It can be used when the polymer must be maintained in solution, or in a further processing step for casting in a mould or, the most interesting, to prepare PAN as fibres or coils using spinning techniques $[6,7,13,14,27,29]$. These fibres would be precursors of carbon fibres after stabilization, precarbonization and carbonization at a temperature of up to 800 $3000^{\circ} \mathrm{C}$ in an inert atmosphere $[5,13,14]$. However, it is difficult to eliminate DMF completely in the final product even after prolonged heating at $110^{\circ} \mathrm{C}$, possibly because of the formation of bonded DMF molecules and molecular interactions with the same PAN, as will be discussed in the next section.

3. Gel processing (monomer is soluble; polymer is not soluble in the solvent): In this case, a different solvent medium (hexane or toluene) facilitates the reaction. Hexane and toluene are solvents of AN, but not of PAN. A previous solution (25 wt.\%) of AN in hexane or toluene was used. The subsequent polymerization yields a gel product containing the insoluble polymer. After heating using temperatures as low as $60^{\circ} \mathrm{C}$, the solvent is completely eliminated, which yields the final product (PAN) in powdered form. This not the case when using DMF, as described above. Furthermore, on a laboratory scale, this processing approach is very suitable because the formation of a compacted product is not a critical factor. It is more useful to prepare a final product with high purity.

4. Foaming processing (neither monomer nor polymer are soluble in the solvent): The selected solvent, considered as a reaction medium, was water. In this case, a mixture of $\mathrm{AN}(25 \mathrm{wt} . \%)$ and deionized water was used after a previous shaking to disperse the AN in small drops to obtain an emulsion. The initiator was incorporated and after polymerization, a white foam mass, which was very porous, was obtained. After heating as low as $60^{\circ} \mathrm{C}$, the water was completely eliminated, yielding a final product (PAN) in powdered form. As in the former approach, the foaming processing is a very convenient method but the effect of 
porosity in the foam is a matter for further investigation. For instance, it was reported that some modified polymers nearly replace the bound water completely, forming a quasi impenetrable adsorbed layer [30]. The water molecule can form simultaneously two hydrogen bonds in this system: one with the $\mathrm{C} \equiv \mathrm{N}$ group of a growing PAN radical and one with a monomer molecule [11].

\subsection{Structural features by XRD}

XRD has been used to investigate the structural differences of the PAN samples prepared according to the processing approaches described above. Fig. 1 shows the X-ray patterns of selected PAN samples. Some differences can be observed between the various PAN samples. First of all, an intense peak at $0.52 \mathrm{~nm}\left(2 \theta=17^{\circ}\right)$ characteristic of PAN is detected in the samples, which is more asymmetric when using the solvents. Secondly, the peak at $0.304 \mathrm{~nm}\left(2 \theta=29^{\circ}\right)$, of a much lower

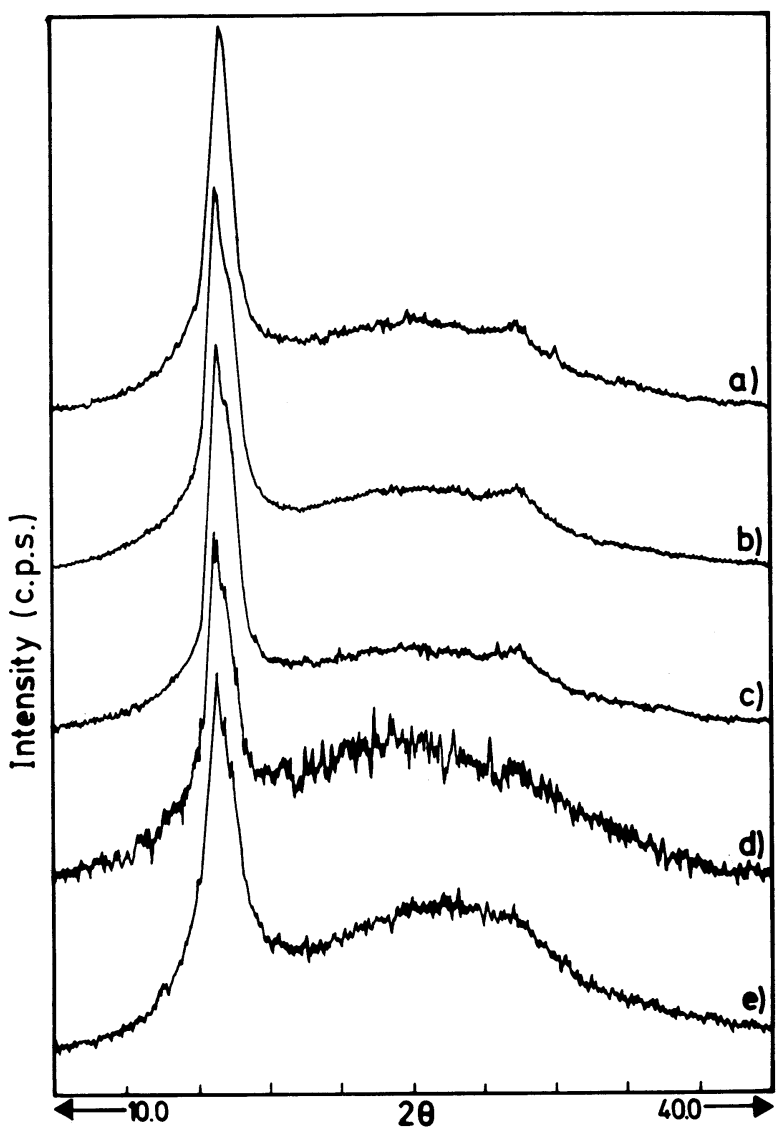

Fig. 1. XRD patterns of PAN samples obtained in bulk form (a), and using DMF (b), hexane (c), toluene (d), and water (e). 
intensity compared with the former, appears only in the PAN obtained when using no solvent, DMF or hexane (Fig. $1 \mathrm{a}-\mathrm{c}$ ). In the other samples, this peak is at the level of the X-ray background or is difficult to detect. According to previous results $[14,18,29]$, PAN fibre with linear chains shows a perfect crystal with a diffraction at $2 \theta=17^{\circ}$ which corresponds to (100) planes, and a minor one at $2 \theta=29^{\circ}$ to (101) planes. According to several authors, it is considered that PAN is a semicrystalline polymer with flexible chains [13,14,31]. Details of the fiber morphology, in the form of an interplay between crystalline and amorphous regions of this polymer, have an essential effect on their thermomechanical properties [32].

On the other hand, a decrease in the widths at half-maximum intensity of the X-ray peaks represents an increase in the crystal size. The measured widths at half-maximum intensity of the first peak (in degrees $2 \theta$ ), which are in relation to the crystal size (X-ray line broadening), suggested that the PAN samples obtained when using water (1.63) and toluene (1.32) as solvents are the samples with a greater content of amorphous components compared to the others (1.22 for PAN obtained using DMF and hexane, and 1.02 for PAN obtained in bulk form). This is related to the crystallinity of the PAN produced under the conditions used in the different processing approaches. For instance, Tsai [29] reported values of these widths (in degrees $2 \theta$ ) ranging from 0.864 to 1.678 as the maximum for the same plane (100) corresponding to PAN precursors of carbon fibre. In other words, the formation of a broad peak at $2 \theta=17^{\circ}$ and a broad and smaller one at $2 \theta=29^{\circ}$ in the X-ray diffraction patterns of some PAN samples (Fig. 1) is associated with a lower order of molecular chains, which exist in a good order of amorphous phase. Finally, it should be noted that PAN fibres have been considered to have a disordered, amorphous, phase as well as an ordered phase $[11,13,14,17]$. The atactic nature of PAN means that the ordered phase cannot be regarded as truly crystalline, but it is thought that the molecular chains have a regular helical configuration owing to strong intermolecular dipole-dipole interactions between nitrile pairs. Bound nitrile group pairs are formed as a result of intermolecular attractions. It is thought that the amorphous phase is composed of irregular helical configurations having less intermolecular nitrile group pairings. Changes in molecular structure of PAN molecules is initiated in the amorphous phase and then forms the boundaries of the ordered phase as reported in the literature [7,17].

\subsection{Thermal study by DSC}

Fig. 2 shows the DSC diagrams of selected PAN samples. In general, broad DSC exotherms can be seen with overlapping peaks, although the PAN sample obtained using DMF showed a single sharp peak. The PAN sample obtained using water showed a similar shape to that observed for the bulk sample (Fig. 2a), which is related to the powdered form finally obtained (Section 3.1). Thus, taking into account these results, it can be deduced that there is a broad fusion range (instead of a narrow zone) where the samples show a thermal change with evolved heat, as a consequence of the heating rate under dynamic conditions. It has been reported [31] that an exothermic reaction ranging between 200 and $350^{\circ} \mathrm{C}$, in an inert 


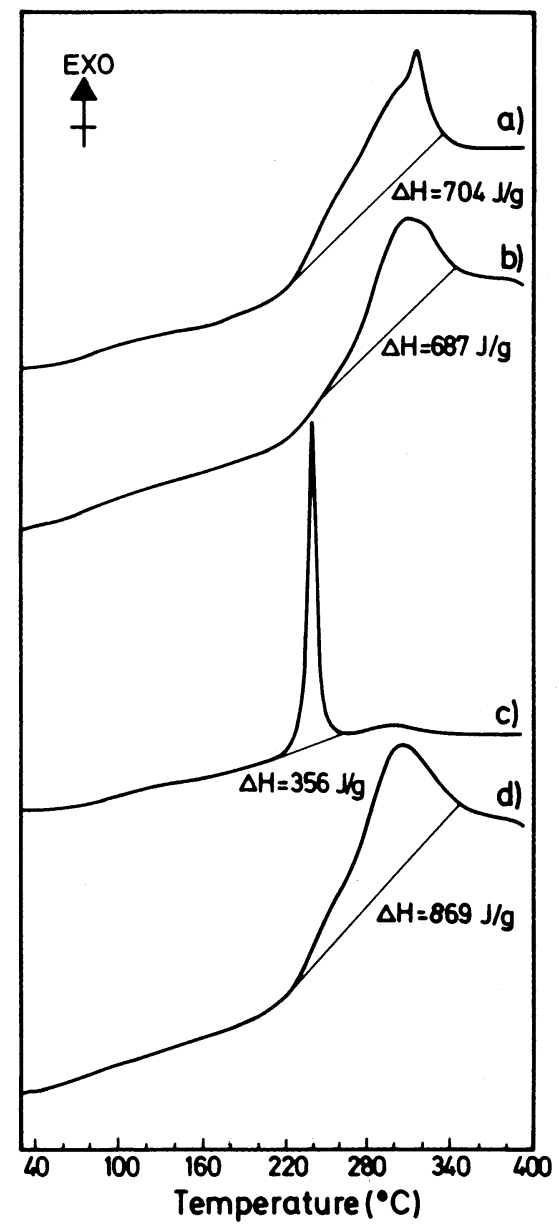

Fig. 2. DSC diagrams of PAN samples obtained in bulk form (a), and using hexane (b), DMF (c), and toluene (d), heating rate $=10^{\circ} \mathrm{C} \min ^{-1}$. Enthalpy results are indicated $\left(\mathrm{J} \mathrm{g}^{-1}\right)$.

atmosphere, is typical of PAN. However, the present DSC runs were performed in air and the effect of this atmosphere on the PAN samples cannot be neglected. Results of onset exotherm peak temperatures, maximum exotherm peak temperatures and evolved heats $\left(\mathrm{J} \mathrm{g}^{-1}\right)$ deduced from DSC diagrams are included in Table 1. The highest heat of exotherm observed for the PAN samples was obtained using toluene, and the lowest using DMF. The onset temperatures are practically the same for all of these samples, with more variation in the maximum peak temperatures, the lowest being for the PAN sample obtained using DMF. It has been reported [11] that the melting temperature for PAN is extremely high $\left(320 \pm 5^{\circ} \mathrm{C}\right)$. Thermodynamic considerations [11,31] suggested that a few degrees of freedom are gained if PAN passes from the crystalline to the molten state, and thus corroborates the view of a stiff, stretched molecule, forced into a helical conformation by steric 
and electrostatic repulsion of the $\mathrm{CN}$ group, even in the molten state. Hence, molecular stiffness may safely be assumed to be the main reason for the high melting point of PAN [11,13,14].

It is remarkable that when DMF is used, this solvent can be considered to exert a plasticizing effect on PAN, as low molecular weight substances offering a high dipole moment, such as amides, as previously pointed out [11] or is even capable of forming 'complexes' with PAN [33]. Moreover, the dominant characteristic of the PAN molecule is the presence of the strongly polar nitrile $(\mathrm{CN})$ groups at an intramolecular distance of only a few tenths of a nanometer. The $\mathrm{CN}$ groups have a variety of ways in which to interact with their surroundings. The lone pair orbital situated at the nitrogen and oriented $180^{\circ}$ away from the $\mathrm{CN}$ bond is perfectly suited to participate in hydrogen bonding with water or other Brönsted acids, as well as in electron-donor-acceptor complex formation with Lewis acids [11]. Thus, molecular interactions of DMF-PAN are expected. Intermolecular dipole-dipole interactions between polymer molecules are replaced by interactions of polymer $\mathrm{CN}$ groups with dipoles of the additive. The presence of the 'plasticizing' compound provides increased mobility of individual segments of the polymer molecules. If all polymer $\mathrm{CN}$ dipoles interact with the dipoles of a liquid, a strongly polar 'additive', dissolution of the polymer is likely (e.g. with DMF or dimethylsulfoxide) [11]. Solutions of salts, such as $\mathrm{NaSCN}$ or $\mathrm{ZnCl}_{2}$, have also been used with success as solvents of PAN [11,27]. It has been reported [14] that plasticized melting studies of PAN fibres using DMF suggested characteristic enthalpy changes associated with first-order transitions, which is in accordance with the sharp DSC peak observed in Fig. 1c when DMF is used for PAN polymerization, establishing the presence of true crystals in the precursor fibres. In fact, in the present study, the addition of DMF is the only case in which both monomer and polymer are soluble in the solvent, as shown in Section 3.1. Thus, DMF is difficult to completely eliminate in the product obtained after the polymerization reaction and even after prolonged heating at $110^{\circ} \mathrm{C}$. DMF molecules remain occluded, possibly by the formation of bonded DMF molecules with the same PAN. In this sense, Keller et al. [33] indicated that DMF molecules form 'complexes' with PAN and the drying of the samples is necessary in order to evaporate all remaining solvent. As a result of this

Table 1

Thermal analysis data of PAN samples

\begin{tabular}{llll}
\hline Sample & $T_{0}{ }^{\mathrm{a}}\left({ }^{\circ} \mathrm{C}\right)$ & $T_{\mathrm{m}}{ }^{\mathrm{b}}\left({ }^{\circ} \mathrm{C}\right)$ & $\Delta H^{\mathrm{c}}\left(\mathrm{J} \mathrm{g}^{-1}\right)$ \\
\hline Bulk (no solvent) & 216 & 311 & 704 \\
Solution (DMF) & 221 & 240 & 356 \\
Solution (hexane) & 219 & 303 & 687 \\
Solution (toluene) & 211 & 296 & 869 \\
\hline
\end{tabular}

a Onset temperature of the DSC peak.

${ }^{\mathrm{b}}$ Maximum temperature of the DSC peak.

${ }^{\mathrm{c}}$ Evolved heat. 
kind of interaction, the DSC diagram is quite different from the other samples studied in the present work. The maximum DSC peak temperature is the lowest $\left(240^{\circ} \mathrm{C}\right)$ of all the samples studied in the present work. A very sharp exothermic peak at ca. $290^{\circ} \mathrm{C}$ was detected [26] for commercially obtained PAN precursors, and associated with a so-called 'violent' exothermic, which shows an onset temperature of $260^{\circ} \mathrm{C}$. Lakeman et al. [27], using DMF for processing PAN fibres, found this peak at a somewhat lower temperature $\left(275^{\circ} \mathrm{C}\right)$, but no heating rate conditions were reported. Fitzer et al. [5], studying homopolymer PAN, observed a sharp DSC peak with a maximum peak temperature of ca. $284^{\circ} \mathrm{C}$, in nitrogen, at a heating rate of $5^{\circ} \mathrm{C} \mathrm{min}^{-1}$ and a broader DSC peak centered at ca. $305^{\circ} \mathrm{C}$, in air, at the same heating rate. It is interesting to note that broad DSC peaks were observed by Fitzer et al. [5] studying copolymer PAN under the same conditions as described above.

Taking into account the above discussion, it can be deduced that the amount of heat evolved as well as the temperature interval over which it is released (exothermic DSC effect), are influenced by the chemical processing of PAN, as described in Section 3.1, in particular when using DMF as solvent for both monomer and polymer. Although it can be considered the melting process when PAN is heated, there is an important step in the mass production of high-performance carbon fibres from PAN: the stabilization process $[5,13,17,31,33]$. The term stabilization is often used to describe the process involved when heating the PAN precursor at $200-300^{\circ} \mathrm{C}$ under controlled conditions in air or in an oxygencontaining atmosphere in order to stabilize it for the succeeding carbonization process and graphitization. This process leads to the formation of a ladder polymer in stabilized fibre. The thermal oxidation process induces dimension stability prior to the carbonization stage by producing an infusible structure. Subsequently, carbonization and graphitization is carried out at higher temperatures in an inert atmosphere in order to obtain the type of carbon fibres desired. When PAN is heated in an inert or oxidizing atmosphere, $\mathrm{C} \equiv \mathrm{N}$ bonds are converted into $\mathrm{C}=\mathrm{N}$ bonds and a cyclization reaction takes place. Thus, the stability in the structure of PAN during oxidation is achieved through the conversion of an open chain structure into a closed chain aromatic structure. These reactions result in the formation of ladder polymers in stabilized fibres. In other words, the thermoplastic chain molecule is transformed into a non-meltable ladder polymer by cyclization, dehydrogenation and oxidation. The mechanistic pathways are described in the next section concerning the pyrolysis reactions of the present PAN samples. The cyclization of PAN during stabilization is always associated with a large exotherm in DSC curves [5,13], as observed in Fig. 2 for the PAN samples processed using different solvents. However, the broadening of the exothermic peaks is a result of the cyclization process modified by the action of the solvent used in the present case for PAN polymerization. It should be noted that the cyclization of the nitrile groups of PAN, which occurs between 250 and $300^{\circ} \mathrm{C}\left(10^{\circ} \mathrm{C} \mathrm{min}{ }^{-1}\right)$, is extremely exothermic, and causes some fragmentation of the chain due to the rapid heat build-up in the sample because the heat does not dissipate at a sufficiently rapid rate [34]. 


\subsection{Thermal study by $P y-G C / M S$}

Conventional pyrolysis has provided valuable information on the thermal degradation of PAN polymers prepared according to the processing approaches described above. Fig. 3 shows the total ion chromatograms of the thermal degradative products obtained after the pyrolysis of the different PAN samples. The main pyrolysis compounds identified in the chromatograms of Fig. 3 are listed in Table 2. The pyrograms of these PAN samples are very similar, although there are some differences. PAN obtained in bulk form has a predominance of nitrile compounds, such as 2-propenitrile (acrylonitrile), peak labeled (1), and 2-methylpropenenitrile (2) and their respective isomers (2,4), and 2-methylene-butanenitrile (5). The presence of 2,4-pentadienenitrile (6) can also be detected in a low relative proportion. Different types of cyclized compounds, such as methylpyridine (10), dimethylpyridine (13) and vinylbenzene (11) appear in the pyrogram. Aromatic nitriles, such as benzonitrile (14) are detected in a relatively high proportion compared to pyridine derivatives, and its methyl derivatives (methyl- and dimethylbenzonitrile) $(15,16)$ which can also be observed. All these compounds could be derived from cyclized PAN structures which are not completely degraded by the thermal treatment of pyrolysis. Finally, alkyldinitriles, such as the compound hexanedinitrile (17), have also been tentatively identified by Py-GC/MS. These kinds of compounds could be formed after the final molecular breakdown of cyclized structures with six-member rings by intensive thermal treatment of pyrolysis.

The same compounds were identified in both the PAN samples obtained using hexane (pyrogram of Fig. 3b) and those identified in bulk PAN. However, when toluene or DMF are used for the preparation of PAN, the pyrograms of the samples (Fig. 3c,d) show the compounds corresponding to PAN thermal degradation, with slight variations in their relative abundance. The peaks corresponding to toluene or DMF also appear in the respective pyrograms. This is especially apparent in the case of the PAN sample obtained using DMF, when a large peak of DMF is released upon Py-GC/MS. This result strongly suggests that this solvent is occluded in the PAN samples and was not eliminated after drying under the present experimental conditions (see Section 3.1). A possible PANDMF complex, as discussed in the preceding section, could be formed in the first step of the processing of PAN using DMF as the solvent medium (both monomer and polymer are soluble in DMF). However, this solvent can be eliminated at relative high temperatures, such as those used in the pyrolysis conditions, which were used to obtain the pyrograms. It is an indication of the strong bond between DMF and PAN, based on polar interactions. Finally, when water is used in the preparation of PAN, the pyrogram of the sample (Fig. 3e) shows the compounds corresponding to PAN thermal degradation, with slight variations in their relative abundance. In general, the peaks are broader compared to those observed for bulk PAN (Fig. 3a). 


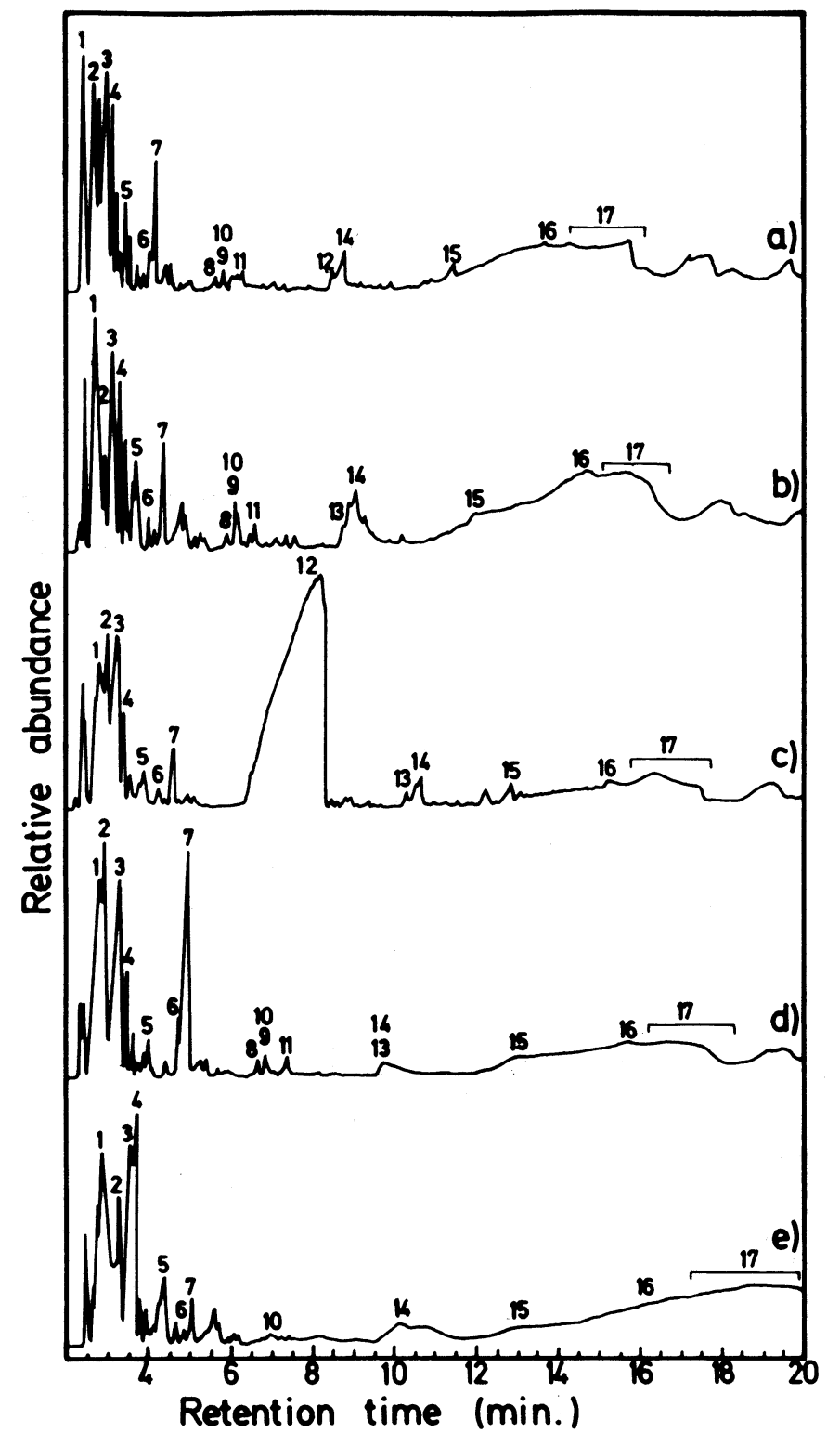

Fig. 3. Total ion chromatograms of the products released in the pyrolysis of PAN samples obtained in bulk form (a), and using hexane (b), DMF (c), toluene (d), and water (e). Numbers refer to the compounds in Table 2 .

\subsection{Thermal process of PAN stabilization and pyrolysis}

It is desirable to have detailed information on the degradation reactions and on the possible mechanism of these reactions during PAN thermal stabilization. 
Thermal degradation techniques are among the oldest approaches used in the study of the structure and degradation patterns of polymers. Thus, thermal analysis by $\mathrm{Py}-\mathrm{GC} / \mathrm{MS}$ is a powerful tool in the structural analysis of the thermal degradation products of different polymers or the same polymer obtained using several processing approaches. The results obtained using Py-GC/MS are further discussed after a summarized description of the thermal features involved in the stabilization process of PAN.

The reaction steps involved in the formation of stabilized PAN (fibres) have been studied and confirmed by several authors $[5,8,13,17,33]$. However, in spite of several studies using various analytical tools, the chemistry of the stabilization of PAN remains complicated and not well understood. Fig. 4 shows the mechanisms suggested for the stabilization chemistry and subsequent pyrolytic carbonization of PAN. Mechanistic pathways were suggested for the thermal degradation of PAN, with the formation of extended two-dimensional structures via condensation of the cyclization products. It is well known that dimension stability of PAN fibres is achieved through the formation of ladder molecular structures generated by the combination of cyclization, dehydrogenation and oxidation reactions. These reactions are predominantly intramolecular and, consequently, only a few cross-links are produced during the thermal reaction process. The cross-links are often referred to as step ladders and arise from strong intermolecular attractions between those

Table 2

Identification of the compounds released in the pyrolysis of PAN samples

\begin{tabular}{ll}
\hline Peak number & Compound \\
\hline 1 & 2-Propenenitrile (acrylonitrile) \\
2 & Isomer of 1 \\
3 & $2-$-Methyl propenenitrile \\
4 & Isomer of 3 \\
5 & 2 -Methyl butenenitrile \\
6 & $2,4-$ Pentadienenitrile \\
7 & Methylbenzene (toluene) \\
8 & Dimethylbenzene (xylene) \\
9 & Isomer of 8 \\
10 & Methylpyridine \\
11 & Vinylbenzene (styrene) \\
12 & $N, N$-Dimethylformamide \\
13 & Dimethylpyridine \\
14 & Benzonitrile \\
15 & Methylbenzonitrile \\
16 & Dimethylbenzonitrile \\
17 & Tentatively identified as an alkyldinitrile \\
\hline
\end{tabular}


(I)

(II)

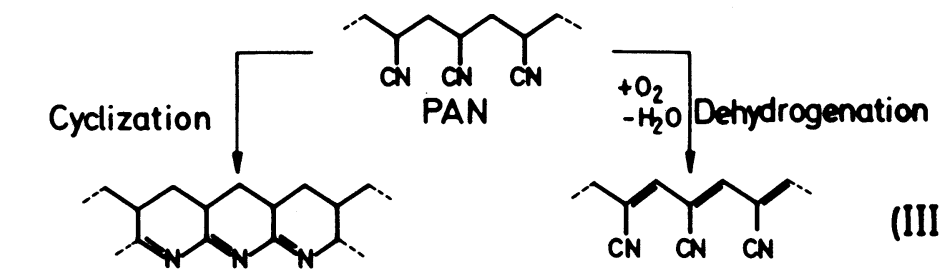

(III)

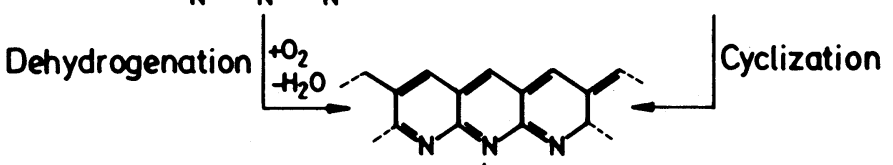

(IV)

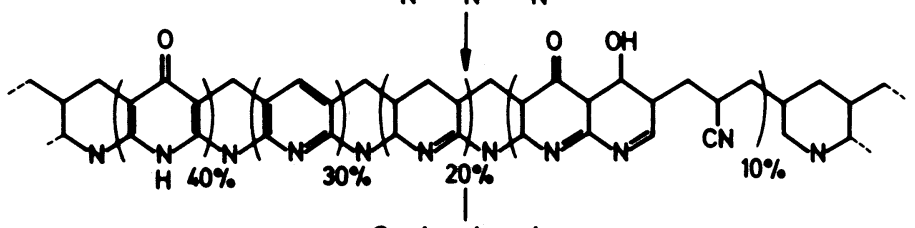

(V)

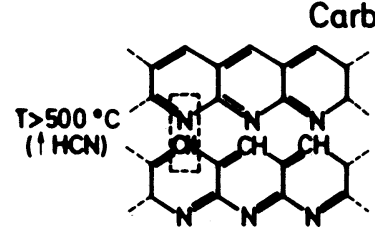

Carbonization

(VI)

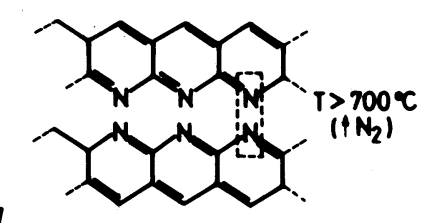

Fig. 4. Mechanistic pathways for the thermal degradation of PAN and reactions involved in the stabilization of PAN fibres, after Fitzer et al. [5] and Fitzer [8].

$\mathrm{CN}$ groups from neighbouring chains, which are 'out-of-step' with the helical configuration along the backbone chains. The step ladder structure is oxidized further and, with the loss of water and the subsequent tautomeric rearrangements, it gives rise to the formation of pyridone units in the chains (Fig. 4, V). It has been reported [5] that the stabilized polymer contains about 8 wt.\% oxygen. The cyclization process for PAN fibres (homopolymer) occurs because of a free radical initiation stage, followed by oxidative reactions. The cyclization of nitrile pendant groups of PAN which give polyimine type structure, seems to be well accepted as the main reaction during stabilization [13]. These imine sequences are postulated to be three to six units long. The presence of polar groups $\mathrm{C}=\mathrm{O}, \mathrm{OH}$ and $\mathrm{COOH}$ in the chains facilitates the initiation of the cyclization process through a nucleophilic attack and accelerates the subsequent oxidative ring closure reactions (Fig. 5). 
Thus, the overall reaction time for the stabilization process is reduced, affording considerable savings in manufacturing costs [17]. This could be the case when DMF is used for the processing of PAN, compared with the other solvents (Fig. 2).

Taking into account the mechanistic pathways (Fig. 4), it is assumed that three main reaction steps take place during the stabilization process of PAN $[5,8,13,17,33]$ :

1. Cyclization of pendant nitrile groups, leading to ladder-type structures, as described above.

2. Formation of conjugated electron systems via dehydrogenation reactions along the polymer backbone and in the condensed ring structures.

3. Oxidation reactions, resulting in the formation of carbonyl and hydroxyl functionalities.

Since the stabilization process in atactic polymers leads only to a partial cyclization and dehydrogenation, a variety of olefinic and aliphatic structure units in aromatic and non-aromatic rings with heteroatoms is expected in the reaction products. It should be noted that the relative abundances (\%) of individual structure units in formula (V) of Fig. 4 are dependent on the stereochemistry of the precursor material and have been reported to vary over a broad range [35].

The detection of exothermic DSC peaks in the range $200-350^{\circ} \mathrm{C}$ can be associated to nitrile group polymerization of PAN, i.e. cyclization, although it is evident that many side reactions, such as the formation of ammonia or even depolymerization, occur in the same temperature region, as reported by Grassie and McGuchan [35]. During subsequent carbonization in a non-oxidizing atmosphere (such as the one used for pyrolysis runs), oxygen and the heterocyclic nitrogen are split off and planar polyaromatics are formed (Fig. 4, VI). All these stabilization and carbonization reactions result in volatile byproducts according to the previous results of Fitzer et al. [5]. Some weight loss from chain scission and elimination of $\mathrm{HCN}$ and $\mathrm{NH}_{3}$ accompanies the cyclization reactions [13]. Oxygen, which is added and is present during stabilization, is mostly present as $-\mathrm{OH}$ and $\mathrm{C}=\mathrm{O}$ groups. However, some authors [36-38] have suggested that oxygen becomes attached to the imine nitrogen atoms to give a nitrone $(\mathrm{N}-\mathrm{O})$ moiety based on infrared spectroscopy and model compound studies. The presence of oxygen promotes crosslinking and helps in the aromatization of the cyclized sequences with the elimination of water, both being essential for the basal plane formation in carbon fibres. In this sense, various mechanisms of thermo-oxidative degradation of PAN polymer have been reviewed

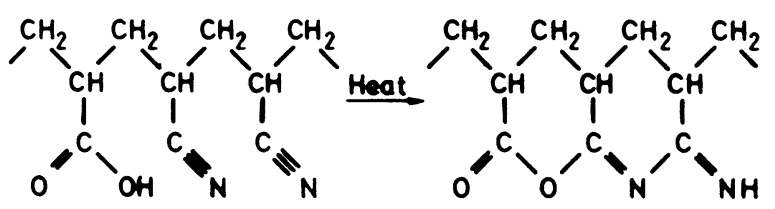

Fig. 5. Molecular model depicting the presence of polar groups $\mathrm{C}=\mathrm{O}, \mathrm{OH}$ and $\mathrm{COOH}$ in the PAN chains which facilitates the initiation of the cyclization process through a nucleophilic attack and accelerates the subsequent oxidative ring closure reactions as shown in Fig. 4. 
by Peebles [39]. The major evolved byproducts formed during the stabilization treatment of PAN in air are $\mathrm{H}_{2} \mathrm{O}, \mathrm{CO}_{2}$ (from dehydrogenation and cross-linking) and $\mathrm{HCN}$. During carbonization, $\mathrm{HCN}$ is also formed as a main volatile byproduct, with the addition of water, ammonia, carbon-oxides and even nitrogen. The evolution of $\mathrm{HCN}$ and $\mathrm{N}_{2}$ is mainly due to the intermolecular condensation of ladder-type structures containing ring nitrogen in large sections. Other pyrolysis products identified during carbonization are $\mathrm{H}_{2}, \mathrm{NH}_{3}, \mathrm{CO}, \mathrm{CH}_{4}$ and $\mathrm{C}_{4}$ hydrocarbons $[13,37,38]$. Elemental nitrogen is formed at the highest carbonization temperatures (Fig. 4, VI). The final carbon fibres (high-temperature carbon fibres) still contain a small percentage of nitrogen (Fig. 4, VII). An important mass loss in PAN is achieved after elimination of all the volatile byproducts.

As far as we know, this is the first time that pyrograms of PAN samples obtained in the laboratory using different solvents, have been reported (Fig. 3). In a previous paper, Usami et al. [40] carried out a structural study of commercial PAN fiber samples (PAN copolymer, with less than a few percent of methyl methacrylate and itaconic acid comonomers), thermally stabilized in air under different conditions. The oxidative thermal degradation of these commercial PAN fibers was studied by Py-GC/MS, ${ }^{13} \mathrm{C}$ NMR and IR spectroscopies. These authors indicated that the contribution of dehydrogenation and/or dehydrocyanide reactions form conjugated polyene structures. Their pyrograms reflected the acrylonitrile sequences in the polymer chain: monomers, dimers, trimers and tetramers. Monomers and oligomeric products were mainly formed through thermal chain scissions along the original polymer chain. As summarized by these authors, the commercial PAN fiber undergoes more or less several types of competitive thermal reactions during the pyrolysis at $700^{\circ} \mathrm{C}$ : (a) cyclization reactions; (b) chain scissions, and (c) dehydrogenation and/or dehydrocyanide with chain scissions and cyclization, with formation of conjugated polyene structures and aromatic compounds (benzenedinitriles and trinitriles). However, their results indicated that the conjugated polyene structures along the commercial PAN fiber molecules should be preliminarily formed, to some extent, during the prolonged stabilization treatment at $235^{\circ} \mathrm{C}$, a remarkable difference with the present results where the starting PAN samples, prepared under laboratory conditions using several processing approaches, have not been previously stabilized (Figs. 2 and 3).

As a result, the competitive thermal reactions suggested by Usami et al. [40] for PAN (copolymer) must be qualitatively distinct according to the present results because the main pyrolysis compounds identified in the chromatograms (Table 2) and those reported by Usami and coworkers show important differences.

\section{Conclusions}

The polymerization of AN has been studied in bulk form and using several solvents: DMF, hexane, toluene, or water. Thermal analyses of the resultant 
products after polymerization have been performed by DSC and Py-GC/MS. XRD has been used to investigate the structural differences. The effect of the solvents employed as media for polymerization is evidenced by the thermal and structural methods. The results described in the present paper will improve our understanding of the evolution of the structure and properties of carbon and activated carbon fibres, which will enable us to establish processing strategies in order to obtain these materials under adequate and reproducible conditions. There is an important difference in crystallinity of the PAN produced under the conditions used in the different processing approaches. Thus, the PAN samples obtained when using water or toluene as solvents have the greater content of amorphous components compared to the other PAN samples obtained using DMF, hexane, or in bulk form (no solvent). The addition of DMF is the only case in which both monomer and polymer are soluble in the solvent. DMF molecules remain occluded, possibly by the formation of bonded DMF molecules with the same PAN. This solvent can be considered to exert a plasticizing effect on PAN or is even capable of forming complexes with this polymer. As a result of this interaction, the DSC diagram is quite different from the other samples studied in the present work, showing a single sharp exothermic peak. This is associated with nitrile group polymerization of PAN, i.e. cyclization, instead of melting. It is deduced that the amount of heat evolved as well as the temperature interval over which it is released (exothermic DSC effect), are influenced by the chemical processing of PAN, in particular when using DMF as solvent for both monomer and polymer.

Pyrolysis of the different PAN samples revealed the release of occluded solvent molecules, mainly when using DMF, and compounds produced from the thermal degradation processes, such as pyridine derivatives and aromatic nitriles. All these compounds could be derived from cyclized PAN structures, which are not completely degraded by the thermal treatment of pyrolysis. Alkyldinitriles have also been tentatively identified associated with the final molecular breakdown of cyclized structures with six-member rings by intensive thermal treatment of pyrolysis. The effect of the solvents employed as media for polymerization is evidenced by the thermal and structural methods. Pyrolysis GC-MS provides valuable complementary information on the structure of the PAN samples (homopolymer) obtained using different processing approaches involving several solvent media. This technique is a powerful tool for the structural analysis of the thermal degradation products of PAN polymers obtained using the different processing approaches described in the present investigation. In addition, this technique will be appropriate for monitoring the changes undergone by the PAN samples during thermal degradation processes involving cyclization and oxidation reactions, which lead to the obtention of stabilized PAN fibres. These changes are different if considering PAN homopolymer, PAN copolymers, and PAN samples which are previously stabilized. The changes of PAN at different stages of the stabilization process are a matter for further investigation along with the objective of providing a more complete picture of this important process in the production of high-performance carbon fibres and activated carbon fibres. 


\section{Acknowledgements}

The authors acknowledge Ana García-Torres for her artwork and Cassandra J. Mooney for manuscript preparation and English review of previous drafts.

\section{References}

[1] O.P. Bahl, L.M. Manocha, Carbon 13 (1974) 297.

[2] G.A. Cooper, R.M. Mayer, J. Mater. Sci. 6 (1971) 60.

[3] R.B. Mathur, Fibre Sci. Technol. 20 (1984) 227.

[4] O.P. Bahl, R.B. Mathur, T.L. Dhami, Mater. Sci. Eng. 73 (1985) 105.

[5] E. Fitzer, W. Frohs, M. Heine, Carbon 24 (1986) 387.

[6] T.H. Ko, H.Y. Tin, C.H. Lin, J. Appl. Polym. Sci. 35 (1988) 631.

[7] T.H. Ko, H.Y. Tin, C.H. Lin, J. Appl. Polym. Sci. 37 (1989) 553.

[8] E. Fitzer, Acta Polym. 41 (1990) 381.

[9] R. Moreton, W. Watt, W. Johnson, Nature 213 (1967) 690.

[10] M. Stewart, M. Feughelman, L.M. Gillin, Nature 235 (1972) 274.

[11] G. Henrici-Olivé, S. Olivé, in: H.J. Cantow, G. Dall'Asta (Eds.), Advances in Polymer Science, vol. 32, Springer, Berlin, 1979, p. 123.

[12] G. Henrici-Olivé, S. Olivé, Adv. Polym. Sci. 51 (1983) 3.

[13] M.K. Jain, A.S. Abhiraman, J. Mater. Sci. 22 (1987) 278.

[14] M.K. Jain, M. Balasubramanian, P. Desai, A.S. Abhiraman, J. Mater. Sci. 22 (1987) 301.

[15] T.H. Ko, S.C. Liu, J. Mater. Sci. Lett. 7 (1988) 628.

[16] D.J. Johnson, in: J.L. Figuereido (Ed.), Carbon Fibres, Filaments and Composites, NATO ASI Series, Kluwer, Dordrecht, 1990, p. 128.

[17] L. Mascia, E.G. Paxton, Thermochim. Acta 184 (1991) 251.

[18] T.H. Ko, L.C. Huang, J. Mater. Sci. 27 (1992) 2429.

[19] T.H. Ko, S.C. Liau, M.F. Lin, J. Mater. Sci. 27 (1992) 6071.

[20] R.Y. Lin, J. Economy, Appl. Polym. Symp. 21 (1973) 143.

[21] R.J. Martin, W.J. Ng, Water Res. 19 (1985) 1527.

[22] T.H. Ko, P. Chiranairadul, C.H. Lin, J. Mater. Sci. Lett. 11 (1992) 6.

[23] K. Kaneko, C. Ishii, M. Ruike, H. Kuwabara, Carbon 30 (1992) 1075.

[24] M.A. Ferro, E. Utrera, J. Rivera, C. Moreno, Carbon 31 (1993) 857.

[25] T.H. Ko, P. Chiranairadul, J. Mater. Res. 10 (1995) 1969.

[26] G. Pan, N. Muto, M. Miyayama, H. Yanagida, J. Mater. Sci. 27 (1992) 3497.

[27] C.D.E. Lakeman, G. Pan, N. Muto, M. Miyayama, H. Yanagida, D.A. Payne, Mater. Lett. 13 (1992) 330.

[28] Y. Sugahara, K. Kuroda, C. Kato, J. Am. Ceram. Soc. 67 (1984) C247.

[29] J.S. Tsai, J. Mater. Sci. Lett. 11 (1992) 140.

[30] E. Volpert, J. Selb, F. Candau, N. Green, J.F. Argillier, A. Audibert, Langmuir 14 (1998) 1870.

[31] L. Sarvaranta, J. Appl. Polym. Sci. 56 (1995) 1085.

[32] D. Eichenauer, H. Jung, Adv. Mater. 4 (1992) 215.

[33] B.A. Keller, C. Löwe, R. Hany, Surface Interface Anal. 22 (1994) 284.

[34] G. Cárdenas, C. Retamal, L.H. Tagle, Thermochim. Acta 188 (1991) 221.

[35] K. Frigge, A. Büchtemann, H.P. Fink, Acta Polym. 42 (1991) 322.

[36] N. Grassie, R. McGuchan, Eur. Polym. J. 6 (1970) 1272.

[37] J.N. Hay, J. Polym. Sci. A-1 6 (1968) 2127.

[38] J.W. Johnson, W.D. Potter, P.G. Rose, G. Scott, Br. Polym. J. 4 (1972) 527.

[39] L.H. Peebles, Jr, Encyclopedia of Chemical Technology, Wiley, New York, 1979, p. 1.

[40] T. Usami, T. Itoh, H. Ohtani, S. Tsuge, Macromolecules 23 (1990) 2460. 